\title{
Stereotactic body radiotherapy for metastatic spinal sarcoma: a detailed patterns-of-failure study
}

\author{
Jonathan E. Leeman, MD, ${ }^{1}$ Mark Bilsky, MD, ${ }^{1}$ llya Laufer, MD, ${ }^{1}$ Michael R. Folkert, MD, PhD, ${ }^{2}$ \\ Neil K. Taunk, MD, MS, ${ }^{1}$ Joseph R. Osborne, MD, PhD, ${ }^{3}$ Julio Arevalo-Perez, MD, ${ }^{3}$ \\ Joan Zatcky, RN, NP, ${ }^{1}$ Kaled M. Alektiar, MD, ${ }^{1}$ Yoshiya Yamada, MD, ${ }^{1}$ and Daniel E. Spratt, MD ${ }^{1,4}$ \\ Departments of ${ }^{1}$ Radiation Oncology and ${ }^{3}$ Radiology, Memorial Sloan Kettering Cancer Center, New York, New York; \\ 2Department of Radiation Oncology, University of Texas Southwestern Medical Center, Dallas, Texas; and ${ }^{4}$ Department of \\ Radiation Oncology, University of Michigan Medical Center, Ann Arbor, Michigan
}

OBJECTIVE The aim of this study was to report the first detailed analysis of patterns of failure within the spinal axis of patients treated with stereotactic body radiotherapy (SBRT) for sarcoma spine metastases.

METHODS Between 2005 and 2012, 88 consecutive patients with metastatic sarcoma were treated with SBRT for 120 spinal lesions. Seventy-one percent of patients were enrolled on prospective institutional protocols. For patients who underwent routine posttreatment total-spine MRI (64 patients, 88 lesions), each site of progression within the entire spinal axis was mapped in relation to the treated lesion. Actuarial rates of local-, adjacent-, and distant-segment failure-free survival (FFS) were calculated using the Kaplan-Meier method.

RESULTS The median follow-up for the cohort was 14.4 months, with $81.7 \%$ of patients followed up until death. The 12-month actuarial rate of local FFS was $85.9 \%$; however, $83.3 \%$ of local failures occurred in conjunction with distantsegment failures. The 12-month actuarial rates of isolated local-, adjacent-, and distant-segment FFS were $98.0 \%$, $97.8 \%$, and $74.7 \%$, respectively. Of patients with any spinal progression $(n=55)$, only $25.5 \%(n=14)$ had progression at a single vertebral level, with $60.0 \%(n=33)$ having progression at $\geq 3$ sites within the spine simultaneously. Linear regression analysis revealed a relationship of decreasing risk of failure with increasing distance from the treated index lesion $\left(R^{2}=0.87\right)$, and $54.1 \%$ of failures occurred $\geq 5$ vertebral levels away. Treatment of the index lesion with a lower biological effective dose (OR 3.2, 95\% Cl 1.1-9.2) and presence of local failure (OR 18.0, 95\% Cl 2.1-152.9) independently predicted for distant spine failure.

CONCLUSIONS Isolated local- and adjacent-segment failures are exceptionally rare for patients with metastatic sarcoma to the spine treated with SBRT, thereby affirming the treatment of the involved level only. The majority of progression within the spinal axis occurs $\geq 5$ vertebral levels away. Thus, total-spine imaging is necessary for surveillance posttreatment.

http://thejns.org/doi/abs/10.3171/2015.11.SPINE151059

KEY WORDS stereotactic body radiotherapy; SBRT; spine metastasis; sarcoma; patterns of failure; oncology

$\mathrm{M}$ ETASTATIC disease to the spine is common, accounting for $70 \%$ of bone metastases and affecting up to $10 \%$ of cancer patients. ${ }^{7,11}$ Spinal metastases can impact quality of life by inflicting neurological compromise and cancer-related pain. Sarcomas are a rare type of tumor that can metastasize to the spine and result in a challenging clinical scenario. Most sarcomas are considered to be insensitive to conventional doses of palliative radiotherapy, resulting in suboptimal control rates. ${ }^{3,4,18}$ Furthermore, there are limited to no effective systemic therapy options for many types of sarcomas. Therefore, treatment of spinal lesions from metastatic sarcoma typically involves surgery and radiotherapy.

Stereotactic body radiotherapy (SBRT) is a modern technique that uses high-dose-per-fraction radiation delivered precisely to the target lesion. SBRT results in lo-

ABBREVIATIONS AFFS = adjacent-segment failure-free survival; DFFS = distant-segment failure-free survival; FA = flip angle; LFFS = local failure-free survival; OS = overall survival; SBRT = stereotactic body radiotherapy.

SUBMITTED September 7, 2015. ACCEPTED November 23, 2015.

INCLUDE WHEN CITING Published online March 4, 2016; DOI: 10.3171/2015.11.SPINE151059. 
cal control rates $>85 \%$, with minimal impact of histology. ${ }^{1,6,10,11,15,19}$ Across a mixture of cancer types, investigators have shown that $83 \%$ of patients with metastatic disease to the spine have multilevel involvement at the time of presentation. ${ }^{5}$ However, because SBRT is a local therapy that targets only the involved lesion, patients are at significant risk of progression at other sites within the spinal axis, which can result in significant morbidity. An ongoing Phase II/III study by the Radiation Therapy Oncology Group, RTOG 0631, which is assessing SBRT for spinal metastases, recommends MRI of only the treated area to assess tumor response (http://www.rtog.org/ClinicalTrials/ProtocolTable/ StudyDetails.aspx?study=0631). There has not, however, been a comprehensive patterns-of-failure study of recurrence within the spinal axis. Such a report could provide support for the type or schedule of follow-up imaging that is optimal following SBRT for spinal metastasis. Herein, we present the first detailed analysis of patterns of spinal failure for patients with metastatic sarcoma to the spine treated with SBRT.

\section{Methods \\ Study Details}

This study was approved by the Memorial Sloan Kettering Cancer Center institutional review board. Consecutive patients with a histologically confirmed diagnosis of high-grade sarcoma metastatic to the spine, who were treated with SBRT at our institution between 2005 and 2012, were identified. Patients with pediatric sarcomas, chordomas, and primitive neuroectodermal/Ewing sarcoma were excluded. For all cases, pathology was centrally reviewed at Memorial Sloan-Kettering Cancer Center, as was all initial and follow-up imaging. This search yielded 88 patients with 120 spinal metastases treated with SBRT, $71 \%$ of whom were enrolled on prospective institutional protocols.

A multidisciplinary team including radiation oncologists, medical oncologists, neurosurgeons, orthopedic surgeons, and neuroradiologists advised the decision to proceed with SBRT. The NOMS (neurologic [N], oncologic $[\mathrm{O}]$, mechanical instability $[\mathrm{M}]$, and systemic disease and medical comorbidities $[\mathrm{S}])^{2,14}$ criteria were applied for all patients presenting with metastatic sarcoma to the spine. Patients were managed with a multidisciplinary approach involving SBRT in conjunction with surgical management and/or interventional techniques including kyphoplasty, when deemed clinically appropriate.

\section{Radiation Therapy}

Stereotactic body radiotherapy techniques were performed as previously reported. ${ }^{16,19}$ Briefly, patients were immobilized using a noninvasive customized cradle. Simulation for treatment planning was performed with the use of CT images with 2-mm slice thickness. The spinal cord and/or cauda equina was contoured with the use of either a myelogram acquired the same day prior to CT simulation or a fused MR image. Planning contours were consistent with International Spine Radiosurgery Consortium consensus guidelines. ${ }^{8}$ Only the involved vertebral level(s) was treated per the Spratt Six Segment recommendations.
Dose constraints have been previously reported. ${ }^{9}$ Seven to 11 coplanar fields (median 8 fields) were set to a single isocenter using dynamic multileaf collimation, with the dose prescribed and normalized to the $100 \%$ isodose line. Image-guided treatments were delivered with the assistance of onboard imaging, including orthogonal kilovoltage imaging and cone-beam CT for every patient. When dose to target volumes was limited by adjacent organs at risk that were appropriate for displacement, an organ displacement method was used in conjunction with interventional radiology, as previously described. ${ }^{13}$

\section{Posttreatment Surveillance}

Patients were seen 2 months after completion of SBRT and every 3 months thereafter. Follow-up included comprehensive physical and neurological examinations and MRI of the spine (CT imaging with or without myelography was used if a contraindication to MRI was present). Nursing follow-up was performed by telephone within 1 week of treatment, and electronic notes were reviewed. Results of any imaging that was performed outside of our institution were acquired and centrally reviewed. Very few of our patients $(<10 \%$ of the cohort) were followed up with regular PET imaging.

For patients who underwent total-spine MRI at our institution, imaging sequences of the spine were acquired with a 1.5-T GE scanner using an 8-channel cervicothoracolumbar surface coil. Acquired sequences included sagittal T1 (FOV 32-36 cm; slice thickness $3 \mathrm{~mm}$; TR 400-650 msec; flip angle [FA] 90 ), sagittal T2 (FOV 32$36 \mathrm{~cm}$; slice thickness $3 \mathrm{~mm}$; TR 3500-4000 msec; FA $90^{\circ}$ ), and sagittal short $\mathrm{T} 1$ inversion recovery (FOV 32-36 $\mathrm{cm}$; slice thickness $3 \mathrm{~mm}$; TR 3500-6000 msec; FA 90). All scans were reviewed by the treating physician and a neuroradiologist.

\section{End Point Definitions and Patterns of Failure Analysis}

Burden of disease was scored according to whether the patient's metastatic disease involved only the treated lesion, was disseminated but limited to bone and spine, or if visceral/extraosseous metastases were present.

Outcomes assessed included local failure-free survival (LFFS), adjacent-segment failure-free survival (AFFS), distant-segment failure-free survival (DFFS), and overall survival (OS). The entire cohort (88 patients) was used to calculate OS (Fig. 1). LFFS calculations incorporated only patients who had posttreatment imaging of the index lesion (82 patients, 106 lesions), because 6 patients died prior to the first follow-up imaging. Patterns-of-failure analysis included only patients with total-spine MRI $(\mathrm{n}=$ 64 patients, 88 lesions). Patients with subtotal-spine imaging or CT imaging were excluded from the patterns-offailure analysis.

Overall survival was defined as the time to death from any cause. LFFS was defined as the time to local failure at the index site treated with SBRT, as determined by a radiographic increase in size of the treated lesion or progression of neurological symptoms that were referable to the treated lesion. AFFS was defined as the time to progression at the adjacent vertebral body, either superior or 


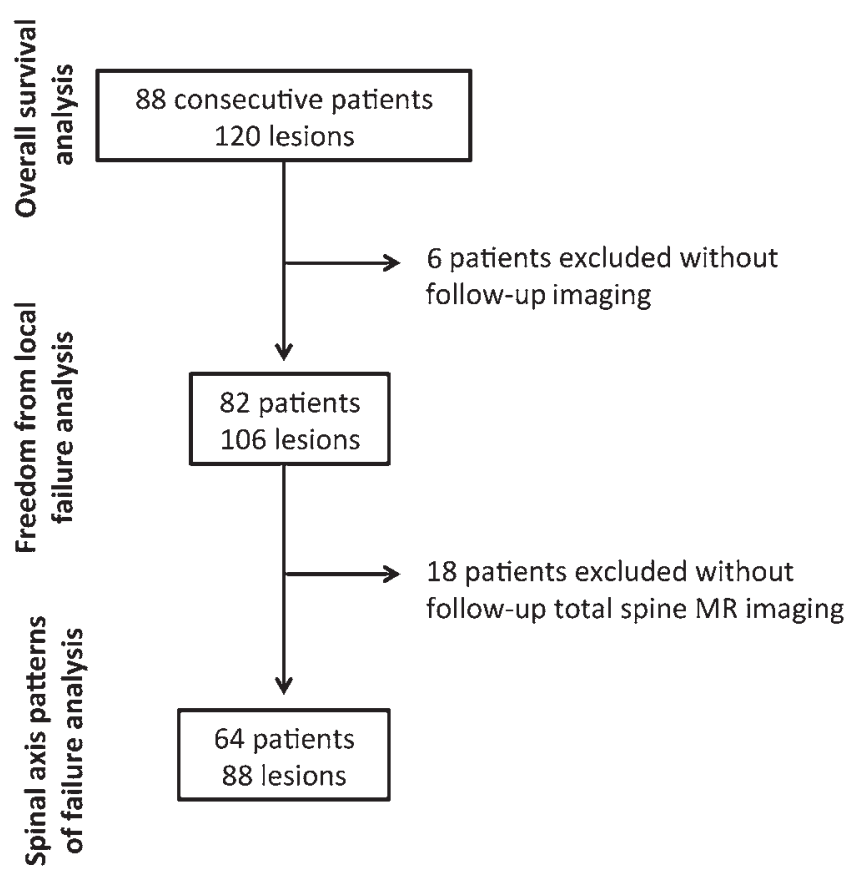

FIG. 1. Study schema.

inferior to the index level. DFFS was defined as the time to any progression along the spinal axis other than local or adjacent failure.

At the time of failure within the spine, the total number of progressive or new lesions within the spine were recorded as well as the distance of each progressive or new lesion from the index treated lesion. These values were used to formulate a recurrence mapping of failures within the spine with respect to the treated vertebral level(s). For the purposes of this analysis, all sacral lesions were considered to be the same level.

For univariate and multivariate analyses, histology was dichotomized by chondrosarcoma and fibrosarcoma versus other histological type. Tumor location was dichotomized by lumbar versus all other locations. The SBRT dose was analyzed as biologically effective dose and was dichotomized to 216 versus < 216 (i.e., 2400 cGy in a single fraction vs other). The planning target volume was analyzed as a continuous variable.

\section{Statistical Analysis}

All outcomes were measured from the date of completion of SBRT to the time of event. Patients were censored at the date of the last follow-up visit or death. Actuarial estimates were performed with Kaplan-Meier statistics. ${ }^{12}$ $\mathrm{R}$-squared statistics were used to assess correlation, and logistic regression modeling was performed for univariate and multivariate analyses. Statistical significance was defined as $\mathrm{p}$ value $\leq 0.05$. Statistical analysis was performed with SPSS, version 22 (SPSS, Inc.).

\section{Results \\ Study Cohort}

The mean age of the cohort was 54 years (range 25-
84 years) and the median Karnofsky Performance Scale score was 90 (range 50-90). Baseline characteristics are shown in Table 1. The median prescribed dose was 2400 cGy (range 1800-3600 cGy) and 56.7\% ( $\mathrm{n}=68)$ of lesions were treated with single-fraction SBRT. The remaining lesions were treated with hypofractionated SBRT (median 3 fractions, range 2-6). At the time of treatment, $71.7 \%$ ( $\mathrm{n}=$ 86) of patients had disseminated disease with visceral metastases. The median follow-up time of the entire cohort was 14.4 months (range $0.6-88.9$ months), with $81.7 \%$ of patients followed up until death. The 12-month actuarial rate of OS was $62.1 \%$, with a median OS of 18.9 months (Fig. 2A).

\section{Patterns of Failure}

Patterns of failure at the time of first failure for patients who underwent follow-up with total-spine MRI are reported in Table 2. Among patients who had progression anywhere in the spinal axis $(\mathrm{n}=55,62.5 \%)$, the majority failed distantly in the spine, with only $5.5 \%(\mathrm{n}=3)$ experiencing isolated local failure and $1.8 \%(\mathrm{n}=1)$ experiencing isolated adjacent-level failure.

The 12-month actuarial rate of LFFS was $85.9 \%$ (Fig. 2B). The 12-month actuarial rate of freedom from any failure within the spine was $57.7 \%$ (Fig. 2C). The 12-month actuarial rates of isolated LFFS (Fig. 3A) and isolated AFFS (Fig. 3B) were $98.0 \%$ and $97.8 \%$, respectively. The 12-month actuarial rate of isolated DFFS ( $\geq 2$ segments from treated index lesion) was $74.7 \%$ (Fig. 3C).

At the time of last follow-up, 37.5\% $(\mathrm{n}=33)$ of cases had not had progression at any site in the spinal axis. Of the remaining $62.5 \%(\mathrm{n}=55)$, only $25.5 \%(\mathrm{n}=14)$ had progression at a single vertebral level, with $60.0 \%(n=33)$ progressing at $\geq 3$ sites within the spine simultaneously (range 0-21 sites, Supplementary Fig. 1).

There was a $7.3 \%$ crude rate of failure at the index lesion site, and rates of failure of $10.9 \%, 11.4 \%, 9.5 \%$, and $6.8 \%$ for lesions $1,2,3$, and 4 segments away, respectively. A total of $54.1 \%$ of failures occurred $\geq 5$ segments away (Fig. 4). Linear regression analysis revealed a high correlation between decreasing risk of failure and increasing distance from the treated index lesion $\left(\mathrm{R}^{2}=0.87\right)$.

Univariate and multivariate logistic regression analyses were performed to identify factors that predict for distant spine recurrence (Supplementary Table 1). Univariate analysis identified histology (OR 5.4, 95\% CI 1.1-25.6; $\mathrm{p}=$ 0.04), biological effective dose (OR 3.2, 95\% CI 1.3-8.2; $\mathrm{p}=0.01)$, and local failure (OR 20.6, 95\% CI 2.6-162.4; $\mathrm{p}$ $<0.01)$ as predictors of distant spine failure. On univariate analysis, extent of disease and presence of epidural disease were not found to be predictors of distant failure. On multivariate analysis, a lower biological effective dose to the index lesion (OR 3.2, 95\% CI 1.1-9.2; $\mathrm{p}=0.03$ ) and presence of local failure (OR 18.0, 95\% CI 2.1-152.9; $\mathrm{p}<$ 0.01 ) independently predicted for higher rates of distant failure.

\section{Discussion}

Understanding the natural history of spread in patients with spine metastases and the patterns of failure 
TABLE 1. Baseline patient and treatment characteristics*

\begin{tabular}{|c|c|}
\hline Variable & Value \\
\hline Total no. of patients & 88 \\
\hline Total no. of treated lesions & 120 \\
\hline Median follow-up time, mos (range) & $14.4(0.6-88.9)$ \\
\hline Mean age, yrs (range) & $54(25-84)$ \\
\hline Median KPS score (range) & $90(50-90)$ \\
\hline \multicolumn{2}{|l|}{ Sex } \\
\hline M & $35(39.8)$ \\
\hline $\mathrm{F}$ & $53(60.2)$ \\
\hline \multicolumn{2}{|l|}{ Surgery per lesion } \\
\hline None for lesion & $82(68.3)$ \\
\hline Before RT & $33(27.5)$ \\
\hline After RT & $5(4.2)$ \\
\hline \multicolumn{2}{|l|}{ Prior RT per lesion } \\
\hline Yes & $12(10)$ \\
\hline None for lesion & $108(90)$ \\
\hline \multicolumn{2}{|l|}{ Histology of lesions } \\
\hline Angiosarcoma & $6(5)$ \\
\hline Chondrosarcoma & $9(7.5)$ \\
\hline Fibrosarcoma & $11(9.2)$ \\
\hline $\begin{array}{l}\text { Hemangiopericytoma/solitary fibrous } \\
\text { tumor }\end{array}$ & $19(15.8)$ \\
\hline Leiomyosarcoma & $36(30)$ \\
\hline Liposarcoma & $17(14.2)$ \\
\hline Other/unspecified spindle cell & $22(18.3)$ \\
\hline \multicolumn{2}{|l|}{ Anatomic distribution of lesions } \\
\hline Cervical & $21(17.5)$ \\
\hline Cervicothoracic & $6(5)$ \\
\hline Thoracic & $51(42.5)$ \\
\hline Thoracolumbar & $3(2.5)$ \\
\hline Lumbar & $27(22.5)$ \\
\hline Lumbosacral & $1(0.8)$ \\
\hline Sacral & $11(9.2)$ \\
\hline Median dose in cGy (range) & $2400(1800-3600)$ \\
\hline \multicolumn{2}{|l|}{ Fractionation per lesion } \\
\hline Single fraction & $68(57)$ \\
\hline Hypofractionated (2-6 fractions) & $52(43)$ \\
\hline Median PTV size in $\mathrm{cm}^{3}$ (mean \pm SD) & $72.0(116.6 \pm 125.4)$ \\
\hline \multicolumn{2}{|l|}{ Epidural involvement prior to treatment } \\
\hline No epidural disease & $62(51.7)$ \\
\hline Epidural disease w/o cord involvement & $40(33.3)$ \\
\hline Cord impingement/abutment & $11(9.2)$ \\
\hline Cord compression & $7(5.8)$ \\
\hline \multicolumn{2}{|l|}{ Extent of disease at time of treatment } \\
\hline Single or solitary spine metastasis & $18(15)$ \\
\hline $\begin{array}{l}\text { Disseminated disease involving spine/ } \\
\text { bone only }\end{array}$ & $16(13)$ \\
\hline Visceral/extraosseous metastases & $86(72)$ \\
\hline
\end{tabular}

KPS = Karnofsky Performance Scale; PTV = planning target volume; RT = radiotherapy.

* Values are reported as number (\%) unless otherwise stated.
TABLE 2. Patterns of failure

\begin{tabular}{lcc}
\hline Type of Progression & No. of Events & $\%$ \\
\hline Local only & 3 & 5.5 \\
\hline Adjacent level only & 1 & 1.8 \\
\hline Distant only & 29 & 52.7 \\
\hline Local \& adjacent & 0 & 0.0 \\
\hline Local \& distant & 6 & 10.9 \\
\hline Adjacent \& distant & 7 & 12.7 \\
\hline Local, adjacent, \& distant & 9 & 16.4 \\
\hline
\end{tabular}

post-SBRT is of critical importance to guide posttreatment surveillance and field design. However, to date, most analyses have investigated tumor and dosimetric variables that predict for local and marginal failure. ${ }^{6,10,17,19}$ To answer the challenging question of patterns of spinal failure, we specifically selected a unique cohort of patients with metastatic sarcoma, for multiple reasons. First, most SBRT spine series involve mixed populations of multiple cancer types. Our study was limited to only patients with high-grade sarcoma to help unify the results. Second, sarcomas were specifically chosen due to the limited efficacy in general of both systemic and targeted therapies for patients with advanced metastatic disease. This reduces the confounding effects of systemic therapies to meaningfully alter the natural history of metastatic spread. This unique cohort of patients has allowed us to provide the first detailed patterns-of-failure analysis, which accounts for the entirety of the spine after SBRT for a spinal metastasis. Additionally, it uses sarcoma, which we believe is an optimal tumor type for such an analysis.

Treatment of spinal metastases with conventional radiation techniques has involved treatment of 1 vertebral level above and 1 vertebral level below the involved segment to ensure adequate target coverage. With the use of $3 \mathrm{D}$ setup verification and the improved precision and dosimetric properties of SBRT, the treatment volume is limited to the involved segment(s) only. ${ }^{8}$ This practice is supported by previous work, which has demonstrated failure rates of $0 \%-5 \%$ in the adjacent vertebral segment after SBRT. ${ }^{10,19}$ Our analysis revealed that the 12-month rate of isolated failure at an adjacent level was only $2.2 \%$. Furthermore, of 17 progression events that we observed at adjacent levels, 16 occurred in conjunction with a distant failure, suggesting the de novo development of a separate metastatic lesion as opposed to a marginal treatment failure. These findings justify treatment of only the involved segment without inclusion of adjacent levels in the target volume.

A previous report from our group described clinical outcomes in this group of patients. Of note, patients who were managed with surgery in combination with SBRT showed similar outcomes to those who were treated nonsurgically. ${ }^{10}$ Patients with surgical hardware at the time of SBRT also showed similar outcomes, with excellent rates of local control. Furthermore, among patients who did develop treatment failures and local progression, $45 \%$ were managed with repeat SBRT, whereas the remainder were treated with surgery, chemotherapy, or best support- 

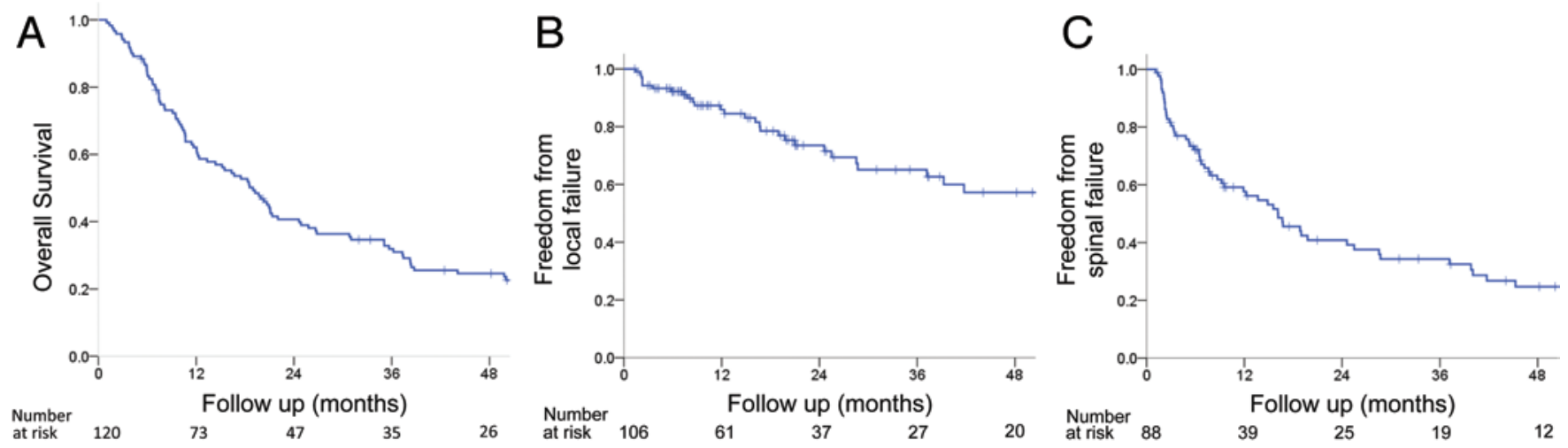

FIG. 2. Actuarial estimated rates of OS (A), LFFS (B), and freedom from any spinal failure (C). Figure is available in color online only.

ive care, depending on the clinical scenario. ${ }^{10}$ Taken together, these data demonstrate that the local therapies for sarcoma spine metastases are effective, and there are multiple available modalities. This highlights the importance of identification of such lesions at an earlier stage and a clear understanding of the patterns of failure throughout the spinal axis.

Our study confirms that local control with spinal SBRT for sarcoma is excellent, thus bringing into question the accuracy of the frequently used term "radioresistant" to describe sarcomas. The 12-month local failure rate was only $13.9 \%$, in keeping with previous reports from our institution and others. ${ }^{1,6,10,11,15,20}$ However, we found that the overwhelming majority of local failures occurred in conjunction with other progression within the spine $(83 \%)$, and the 12-month isolated local failure rate was only $2 \%$. This pattern of multifocal failures throughout the spine may reflect transformation of the disease to a more aggressive phenotype.

The ongoing RTOG 0631 Phase II/III study is assessing SBRT for spinal metastases and currently recommends MRI of only the treated area to assess tumor response (http://www.rtog.org/ClinicalTrials/ProtocolTable/ StudyDetails.aspx?study=0631). Our data suggest a close linear relationship between decreasing risk of failure and increasing distance from the treated index lesion $\left(\mathrm{R}^{2}=\right.$ 0.87). This may be an indicator of a possible common blood supply of involved vertebral levels within the same region of the spine. However, our data demonstrate that at the time of spinal progression, $60.0 \%$ of patients were found to have progression at $\geq 3$ sites within the spine simultaneously. Furthermore, we found that $54.1 \%$ of failures occurred $\geq 5$ segments away from the treated lesion. Our data indicate the importance of total-spine imaging rather than focused spine imaging at regular intervals because these patients are at high risk for failure at distant levels within the spine. Regular imaging is important because detection of spinal metastases at an early stage is critical for mitigation of potential morbidity due to neurological involvement, which can be devastating for patients. Furthermore, optimal dosimetry with SBRT can be achieved more readily when lesions are discovered at a stage when there is absent or mild epidural disease as opposed to after patients have developed high-grade cord compression.

It has been shown in other cancer types that metastatic lesions can be the nidus of further metastatic deposits. Our multivariate regression analysis demonstrated that improved local control was the most predictive factor for distant spine failure. Local eradication may prevent further dissemination within the spine by eliminating sites of disease that could serve as sources for further spread of spinal metastases. These data further highlight the importance of achieving tumor control, not just for alleviation
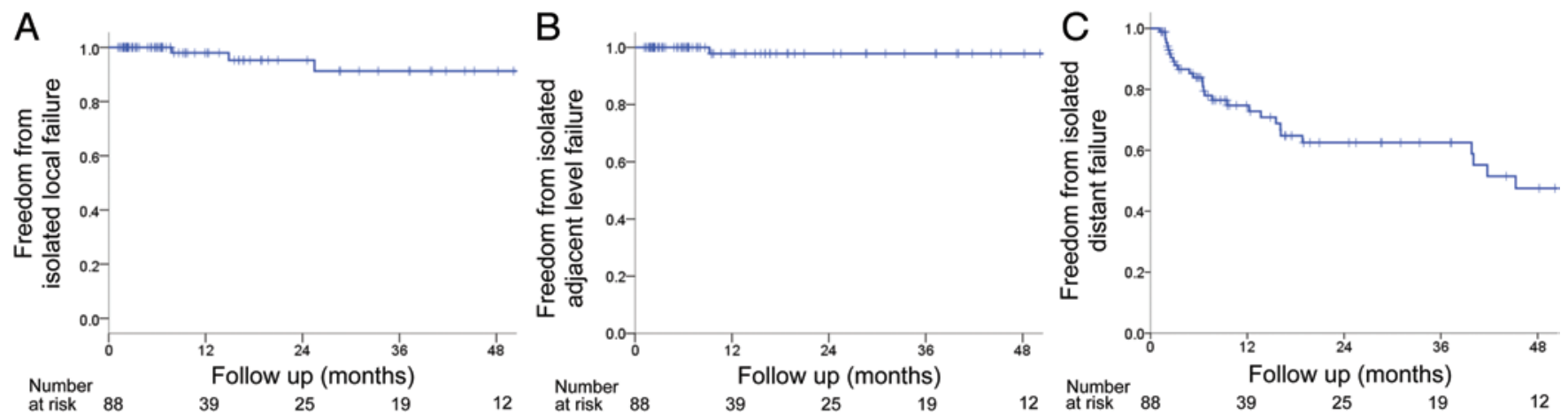

FIG. 3. Actuarial estimated rates of isolated LFFS (A), isolated AFFS (B), and isolated DFFS (C). Figure is available in color online only. 


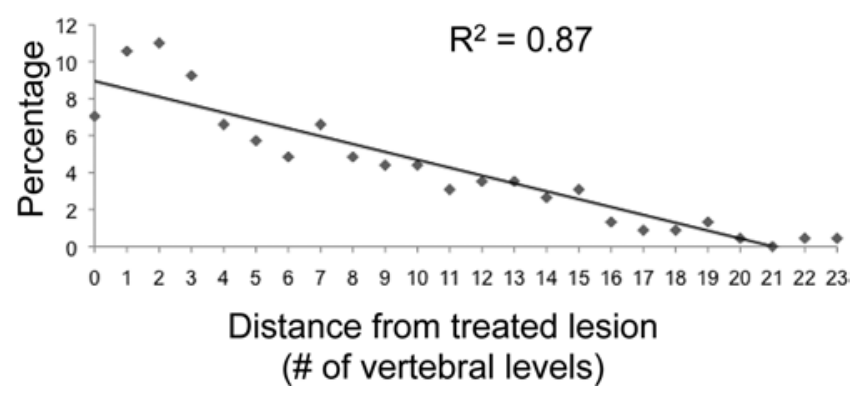

FIG. 4. Distribution of spinal failures in relation to the distance from the lesion treated with SBRT.

and prevention of symptoms, but perhaps also for limiting further metastasis and preventing an increase in disease burden, although this is an area that warrants further investigation. Additionally, lower biologically effective dose to the index lesion was predictive of distant spine failure. Currently, the optimal treatment dose and fractionation are not known. To address this question, multiple randomized trials are ongoing (https://clinicaltrials.gov; NCT00922974 and NCT01223248).

Our study is limited by the retrospective methodology and associated biases. By only including metastases from sarcoma primary tumors, our patterns-of-failure analysis may be less generalizable to other histological types where systemic options play a larger role in the metastatic setting. Our patient population generally had advanced disease at the time of treatment, with $72 \%$ harboring visceral/ extraosseous metastases. Therefore, the failure rates that we observed may be less applicable to patients with a low burden of disease in the oligometastatic setting, although these are generally the minority of patients.

\section{Conclusions}

Isolated local- and adjacent-segment failures are exceptionally rare for patients with metastatic sarcoma to the spine treated with SBRT, thereby affirming the treatment of the involved level only. Patients treated with SBRT for spinal metastases are at high risk of failure throughout the spinal axis. These data highlight the importance of close interval imaging with total-spine MRI. Due to the high rate of distant spine failure, patient selection is critical. Further work is necessary to better identify patients who would benefit long term from spine SBRT. Ongoing clinical trials aim to address this need.

\section{References}

1. Amdur RJ, Bennett J, Olivier K, Wallace A, Morris CG, Liu $\mathrm{C}$, et al: A prospective, phase II study demonstrating the potential value and limitation of radiosurgery for spine metastases. Am J Clin Oncol 32:515-520, 2009

2. Bilsky M, Smith M: Surgical approach to epidural spinal cord compression. Hematol Oncol Clin North Am 20:13071317, 2006

3. Bilsky MH, Gerszten P, Laufer I, Yamada Y: Radiation for primary spine tumors. Neurosurg Clin N Am 19:119-123, 2008

4. Boriani S, Saravanja D, Yamada Y, Varga PP, Biagini R, Fisher CG: Challenges of local recurrence and cure in low grade malignant tumors of the spine. Spine (Phila Pa 1976) 34 (22 Suppl):S48-S57, 2009

5. Botelho RV, de Oliveira MF, Rotta JM: Quantification of vertebral involvement in metastatic spinal disease. Open Orthop J 7:286-291, 2013

6. Chang EL, Shiu AS, Mendel E, Mathews LA, Mahajan A, Allen PK, et al: Phase I/II study of stereotactic body radiotherapy for spinal metastasis and its pattern of failure. J Neurosurg Spine 7:151-160, 2007

7. Chao ST, Koyfman SA, Woody N, Angelov L, Soeder SL, Reddy CA, et al: Recursive partitioning analysis index is predictive for overall survival in patients undergoing spine stereotactic body radiation therapy for spinal metastases. Int J Radiat Oncol Biol Phys 82:1738-1743, 2012

8. Cox BW, Spratt DE, Lovelock M, Bilsky MH, Lis E, Ryu S, et al: International Spine Radiosurgery Consortium consensus guidelines for target volume definition in spinal stereotactic radiosurgery. Int J Radiat Oncol Biol Phys 83:e597e605, 2012

9. Folkert MR, Bilsky MH, Tom AK, Oh JH, Alektiar KM, Laufer I, et al: Outcomes and toxicity for hypofractionated and single-fraction image-guided stereotactic radiosurgery for sarcomas metastasizing to the spine. Int J Radiat Oncol Biol Phys 88:1085-1091, 2014

10. Gerszten PC, Burton SA, Ozhasoglu C, Welch WC: Radiosurgery for spinal metastases: clinical experience in 500 cases from a single institution. Spine (Phila Pa 1976) 32:193-199, 2007

11. Gerszten PC, Mendel E, Yamada Y: Radiotherapy and radiosurgery for metastatic spine disease: what are the options, indications, and outcomes? Spine (Phila Pa 1976) 34 (22 Suppl):S78-S92, 2009

12. Kaplan EL, Meier P: Nonparametric estimation from incomplete observations. J Am Stat Assoc 53:457-481, 1958

13. Katsoulakis E, Solomon SB, Maybody M, Housman D, Niyazov G, Riaz N, et al: Temporary organ displacement coupled with image-guided, intensity-modulated radiotherapy for paraspinal tumors. Radiat Oncol 8:150, 2013

14. Laufer I, Rubin DG, Lis E, Cox BW, Stubblefield MD, Yamada Y, et al: The NOMS framework: approach to the treatment of spinal metastatic tumors. Oncologist 18:744-751, 2013

15. Levine AM, Coleman C, Horasek S: Stereotactic radiosurgery for the treatment of primary sarcomas and sarcoma metastases of the spine. Neurosurgery 64 (2 Suppl):A54-A59, 2009

16. Lovelock DM, Hua C, Wang P, Hunt M, Fournier-Bidoz N, Yenice K, et al: Accurate setup of paraspinal patients using a noninvasive patient immobilization cradle and portal imaging. Med Phys 32:2606-2614, 2005

17. Lovelock DM, Zhang Z, Jackson A, Keam J, Bekelman J, Bilsky M, et al: Correlation of local failure with measures of dose insufficiency in the high-dose single-fraction treatment of bony metastases. Int J Radiat Oncol Biol Phys 77:12821287,2010

18. Merimsky O, Kollender Y, Bokstein F, Issakov J, Flusser G, Inbar MJ, et al: Radiotherapy for spinal cord compression in patients with soft-tissue sarcoma. Int J Radiat Oncol Biol Phys 58:1468-1473, 2004

19. Ryu S, Rock J, Rosenblum M, Kim JH: Patterns of failure after single-dose radiosurgery for spinal metastasis. J Neurosurg 101 (Suppl 3):402-405, 2004

20. Yamada Y, Bilsky MH, Lovelock DM, Venkatraman ES, Toner S, Johnson J, et al: High-dose, single-fraction imageguided intensity-modulated radiotherapy for metastatic spinal lesions. Int J Radiat Oncol Biol Phys 71:484-490, 2008

\section{Disclosures}

Dr. Yamada is a consultant for Varian Medical Systems, Inc., 
and a member of the Speakers Bureau for the Institute for Medical Education. Dr. Laufer is a consultant for Spine Wave, DePuy Synthes, and Globus.

\section{Author Contributions}

Conception and design: Leeman, Bilsky, Laufer, Folkert, Taunk, Osborne, Zatcky, Alektiar, Yamada, Spratt. Acquisition of data: Leeman, Bilsky, Laufer, Folkert, Taunk, Arevalo-Perez, Zatcky, Alektiar, Yamada, Spratt. Analysis and interpretation of data: all authors. Drafting the article: Leeman, Bilsky, Laufer, Folkert, Arevalo-Perez, Yamada, Spratt. Critically revising the article: all authors. Reviewed submitted version of manuscript: all authors. Approved the final version of the manuscript on behalf of all authors: Leeman. Statistical analysis: Leeman, Yamada, Spratt. Administrative/technical/material support: Leeman, Bilsky,
Laufer, Arevalo-Perez, Yamada, Spratt. Study supervision: Leeman, Bilsky, Laufer, Folkert, Osborne, Arevalo-Perez, Zatcky, Alektiar, Yamada, Spratt.

\section{Supplemental Information}

Online-Only Content

Supplemental material is available with the online version of the article.

Supplementary Figure and Table. http://thejns.org/doi/ suppl/10.3171/2015.11.SPINE151059.

\section{Correspondence}

Jonathan E. Leeman, Department of Radiation Oncology, Memorial Sloan Kettering Cancer Center, 1275 York Ave., New York, NY 10065. email: leemanj@mskcc.org. 